\title{
Electronic and magnetic structure of $\mathrm{La}_{0.875} \mathrm{Sr}_{0.125} \mathrm{MnO}_{3}$ calculated by means of hybrid density-functional theory
}

\author{
Sergei Piskunov, ${ }^{1,2, *}$ Eckhard Spohr, ${ }^{1}$ Timo Jacob ${ }^{3}$ Eugene A. Kotomin,,${ }^{2,4}$ and Donald E. Ellis ${ }^{5}$ \\ ${ }^{1}$ Forschungszentrum Jülich, IEF-3, D-52425 Jülich, Germany \\ ${ }^{2}$ Institute of Solid State Physics, University of Latvia, 8 Kengaraga Street, Riga LV-1063, Latvia \\ ${ }^{3}$ Fritz-Haber-Institut der Max-Planck-Gesellschaft, Faradayweg 4-6, D-14195 Berlin, Germany \\ ${ }^{4}$ European Commission Institute for Transuranium Elements, Joint Research Center, D-76125 Karlsruhe, Germany \\ ${ }^{5}$ Department of Physics and Astronomy, Northwestern University, Evanston, Illinois 60208-3108, USA
}

(Received 3 July 2007; published 30 July 2007)

\begin{abstract}
We present the results of ab initio calculations on magnetic and electronic structures of $\mathrm{La}_{1-x} \mathrm{Sr}_{x} \mathrm{MnO}_{3}$ at low doping, $x=1 / 8$. Using the B3LYP hybrid exchange-correlation functional within the framework of densityfunctional theory, we predict a ferromagnetic ground state for $\mathrm{La}_{0.875} \mathrm{Sr}_{0.125} \mathrm{MnO}_{3}$ in both the low-temperature orthorhombic and the high-temperature pseudocubic phases. This is in contrast to its parent compound $\mathrm{LaMnO}_{3}$, for which we find in agreement with experiment the layered antiferromagnetic state to be the most stable one. The calculated density of states and bond population analysis suggest a tendency of formation of half-metallic spin states in the band gap of both structures.
\end{abstract}

DOI: 10.1103/PhysRevB.76.012410

PACS number(s): 75.40.Mg, 71.15.Nc

Sr-doped $\mathrm{LaMnO}_{3}\left(\mathrm{La}_{1-x} \mathrm{Sr}_{x} \mathrm{MnO}_{3}, \mathrm{LSM}\right)$ is one of many extensively studied perovskite-type oxides, attractive due to its colossal magnetoresistivity, a spin-glass behavior, a metal-to-insulator transition [at $\sim 360 \mathrm{~K}$ for $x=0.3$ (Ref. 1)], charge ordering after doping with $\mathrm{Sr}$, and a ferromagneticantiferromagnetic transitions observed. One of its important applications is also the use as a promising cathode material in high-temperature solid oxide fuel cells (SOFCs). ${ }^{2}$ The high operating temperature of current SOFC technology is mandated by the necessity of high oxygen molecule reduction rate at the fuel cell cathode and high oxygen ion mobility in the solid electrolyte and in the cathode bulk or on surfaces and grain boundaries. Technologically, the first requirement (and partially the second one) can be met by reducing the thickness of electrolyte and cathode layers, as it is implemented in the Jülich planar substrate SOFC concept, where thin electrolyte (yttria stabilized zirconium oxide) and cathode layers (LSM or $\{\mathrm{Co}, \mathrm{Fe}\}$-doped $\mathrm{LaMnO}_{3}$ ) are supported by a millimeter thick nickel cermet anode. ${ }^{3}$ Elucidating the mechanisms of oxygen reduction and mobility on LSM surfaces and in the bulk of LSM is thus beneficial for understanding the limitations of the currently used materials in SOFC technology and secondly aiding the development of higher-performance materials.

LSM compounds exhibit a large variety of properties depending on the $\mathrm{La} / \mathrm{Sr}$ ratio, the degree of oxygen nonstoichiometry, temperature and/or pressure conditions, sample preparation, etc. Even at high SOFC working temperatures $(T>780 \mathrm{~K})$, when cooperative Jahn-Teller distortion disappears, magnetic orbital ordering still can take place. Knowledge of proper spin redistribution in manganites is especially important because of the four unpaired electrons on Mn, which demand open-shell calculations. Thus, before one starts modeling the complex surface processes, it is indispensable to first investigate the magnetic and electronic structure of bulk LSM. In the current study, we chose to investigate the LSM with a $12.5 \% \mathrm{Sr}$ doping, which is somewhat larger than typical experimental concentrations $(\leqslant 10 \%$
$\mathrm{Sr}$ ) in order to simplify the calculations (the larger the $\mathrm{Sr}$ content, the smaller the necessary system size).

Recent series of local spin-density approximation calculations performed on LSM were devoted to magnetic ordering with respect to lattice distortion, ${ }^{4}$ the distribution of $\mathrm{Mn}^{3+}$ and $\mathrm{Mn}^{4+}$ charge ordering, ${ }^{5}$ and the surface ferromagnetism. ${ }^{6}$ Experimentally, the nature of the chemical bonds, magnetic properties, metal-insulator transitions, and structural LSM transformations in the vicinity of $x=1 / 8$ were studied in Refs. 7-9.

Recent studies have shown that hybrid exchangecorrelation functionals can accurately reproduce the basic bulk and surface properties of a number of $A_{B} \mathrm{O}_{3}$ perovskite materials, ${ }^{10,11}$ including the low-temperature phase of $\mathrm{LaMnO}_{3}$ (LMO). ${ }^{12,13}$ We adopted here the hybrid B3LYP exchange-correlation functional within the density-functional theory (DFT), following Refs. 12 and 13. The simulations were carried out with the CRYSTAL computer code, ${ }^{14}$ which employs atom-centered Gaussian-type functions as basis sets (BSs).

The BSs used in this study for both LSM and LMO bulk computations were taken from the following sources: for $\mathrm{Sr}$ and $\mathrm{O}$ in the form of $311 \mathrm{~d} 1 \mathrm{G}$ and $8-411 \mathrm{~d} 1 \mathrm{G}$, respectively, from Ref. 10, for $\mathrm{Mn}$ and $\mathrm{La}$ in the form of $86-411 \mathrm{~d} 41 \mathrm{G}$ from Ref. 15, and 311-31d3f1 from CRYSTAL's homepage, ${ }^{14}$ respectively. For $\mathrm{Mn}$ and $\mathrm{O}$, all electrons are explicitly included. The inner core electrons of $\mathrm{Sr}$ and $\mathrm{La}$ are described by small-core Hay-Wadt effective pseudopotentials ${ }^{16}$ and by the nonrelativistic pseudopotential of Dolg et al. ${ }^{17}$ respectively. The choice of BS follows the detailed basis set analysis in Ref. 13, and thus gives us confidence on the reliability of the present calculations. In case of La, we also added an $f$-type polarization Gaussian function with exponent optimized in the LMO crystalline field $(\alpha=0.475)$ with only minor impact on the results, in agreement with the results in Ref. 5. The total energy is lowered by only $1.2 \mathrm{eV}$ per $40-$ atom unit cell and $f$ orbital population does not exceed $0.01 e$. 
The reciprocal space integration was performed by sampling the Brillouin zone with the $4 \times 4 \times 4$ Pack-Monkhorst mesh $^{18}$ (36 $k$ points in total). The cutoff threshold parameters of CRYSTAL for Coulomb and exchange integrals evaluation (ITOL1-ITOL5) have been set to 8, 8, 8, 8, and 16, respectively. Calculations were considered as converged only when the total energy obtained in the self-consistency procedure differs by less than $10^{-7}$ a.u. in two successive cycles.

In this Brief Report, we consider LSM in its lowtemperature orthorhombic phase as well as in its hightemperature (pseudo)cubic phase which is thermodynamically stable at SOFC working conditions. ${ }^{19}$ In order to verify our approach, we also performed a number of test calculations on LMO in its low-temperature orthorhombic structure, which is the parent compound for LSM and well studied by various experiments and calculations. ${ }^{12,13,20-22}$

To model LSM at low doping $(x=1 / 8)$ in the lowtemperature regime (orthorhombic Pbnm phase), we used structural parameters derived experimentally by means of neutron diffraction. ${ }^{23}$ This orthorhombic structure of $\mathrm{ABO}_{3}$ perovskite comprises four formula units. Therefore, to obtain the desired concentration of Sr in LSM, we doubled the initial LMO orthorhombic unit cell, and then one of the eight La atoms was substituted for Sr. Such a doubled orthorhombic unit cell contains 40 atoms in total and exhibits no point group symmetry. We applied the same approach to perform a comparative simulation on undoped LMO. Structural parameters for the low-temperature orthorhombic Pbnm phase of LMO have been taken from the experimental neutron diffraction study. ${ }^{21}$ The experimentally defined geometries have been used in our first-principles calculations, because magnetic properties, especially the coupling constants $J$, are strongly affected by the choice of crystal structure. At the same time, we note that lattice parameters and atomic coordinates of both LMO and LSM optimized by means of the B3LYP hybrid technique are very close to the experimentally observed ones. ${ }^{21}$ Thus, using experimental geometries we expect negligible changes in calculated total energies and in the physical nature of magnetic and electronic structures.

At high temperatures $(T \gtrsim 800 \mathrm{~K})$, the orthorhombic LSM lattice becomes metrically cubic. ${ }^{19}$ We used a $2 \times 2 \times 2$ supercell (40 atoms) of the $P m \overline{3} m$ cubic structure of LMO as a starting geometry. A $\mathrm{Sr}$ concentration of $x=1 / 8$ has been achieved by replacing one of the eight La by Sr. Full geometry (lattice constant and atomic coordinates) optimization was performed in the $P m \overline{3} m$ symmetry. In order to describe orbital ordering for the studied magnetic configurations, the imposed cubic symmetry has been artificially removed.

We have considered four possible magnetic states for both LSM and LMO: the fully ferromagnetic (FM) system, an orthorhombic (cubic) structure with ferromagnetic Mn spin coupling in the basal $a b(x y)$ plane and antiferromagnetic coupling along the $c(z)$ direction (A-type AFM), antiferromagnetic coupling in the basal plane and ferromagnetic coupling along the $z$ direction $(C$-AFM), and antiferromagnetic coupling between all nearest neighbor spins $(G$-AFM). According to Hund's rule, the lowest energy corresponds to the maximal spin projection on the $\mathrm{Mn}^{3+}$ ion $\left(S_{z}=2\right.$, four spin-up $d$ electrons occupy $t_{2 g}$ and $e_{g}$ levels). The energy of each
TABLE I. The energy difference of three considered antiferromagnetic structures of low-temperature LMO (orthorhombic phase) and both low-temperature (orthorhombic phase) and hightemperature (pseudocubic) LSM with respect to their FM solutions. The values are in $\mathrm{meV}$ per 40-atom supercell, which contains $8 \mathrm{Mn}$ atoms.

\begin{tabular}{lccc}
\hline \hline Material & $A$-AFM & $C$-AFM & $G$-AFM \\
\hline LMO, orthorhombic & -65 & 94 & 54 \\
LSM, orthorhombic & 468 & 499 & 1329 \\
LSM, pseudocubic & 1105 & 1386 & 2491 \\
\hline \hline
\end{tabular}

magnetic state has been calculated for all structures using the spin-polarized B3LYP hybrid DFT approach (Table I). For each system, several initial spin distributions have been used to ensure that we found the correct ground state configuration. In agreement with experiment ${ }^{20}$ and recent $a b$ initio calculations, ${ }^{12,13}$ the orthorhombic $A$-AFM structure is identified as the LMO ground state. For the low-temperature phase of slightly doped LSM, the calculated ferromagnetic order is also observed experimentally. ${ }^{7}$ Our calculations suggest that the ferromagnetic ordering of LSM could survive even at SOFC working temperatures, since the energetically favorable pseudocubic high-temperature LSM structure is also ferromagnetic.

In order to compute the relevant magnetic coupling constants (exchange integrals) from the calculated total energies of the magnetic structures, we used the Ising model Hamiltonian

$$
H=-J_{1} \sum_{i j} S_{z i} S_{z j}-J_{2} \sum_{k l} S_{z k} S_{z l},
$$

where $J_{1}$ and $J_{2}$ are magnetic coupling constants between nearest Mn centers in the $x y$ (basal) plane and between nearest centers along the $z$ axis, respectively. $S_{z i}$ stands for the $z$ component of total spin on the magnetic center $i$, and $i j$ and $\mathrm{kl}$ indicate summation over intraplane and interplane nearest magnetic centers, respectively. Positive (negative) values of magnetic coupling constant indicate ferromagnetic (antiferromagnetic) interaction. Following Ref. 24, the following overdetermined set of equations relates the energy differences to the magnetic coupling constants:

$$
\begin{gathered}
E_{\mathrm{tot}}^{\mathrm{FM}}-E_{\mathrm{tot}}^{A-\mathrm{AFM}}=-64 J_{2}, \\
E_{\mathrm{tot}}^{G-\mathrm{AFM}}-E_{\mathrm{tot}}^{\mathrm{FM}}=128 J_{1}+64 J_{2}, \\
E_{\mathrm{tot}}^{C-\mathrm{AFM}}-E_{\mathrm{tot}}^{\mathrm{FM}}=128 J_{1} .
\end{gathered}
$$

These equations are written for a supercell containing eight Mn magnetic centers. Calculated coupling constants are summarized in Table II. In the case of the orthorhombic LMO, the calculated magnetic coupling constants agree well with experiment, suggesting that the chosen calculation scheme is sufficiently reliable. (The analysis ${ }^{13}$ of existing numerous calculations of the LMO coupling constants shows that our agreement within accuracy of a factor 2 could be considered as a good one.) We thus expect our LSM results 
TABLE II. The magnetic coupling constants $J_{1}$ and $J_{2}$, in meV, for low-temperature LMO (orthorhombic phase) and both lowtemperature (orthorhombic phase) and high-temperature (pseudocubic) LSM calculated as average from the overdetermined set of equations (2)-(4) and using the energies of magnetic phases reported in Table I.

\begin{tabular}{lcc}
\hline \hline Material & $J_{1}$ & $J_{2}$ \\
\hline LMO, experiment $^{\mathrm{a}}$ & $1.6^{\mathrm{b}}$ & -1.2 \\
LMO, experiment $^{\mathrm{c}}$ & 1.0 & -1.2 \\
LMO, orthorhombic & $0.8 \pm 0.1$ & $-0.8 \pm 0.2$ \\
LSM, orthorhombic & $5.3 \pm 1.4$ & $10.1 \pm 2.8$ \\
LSM, pseudocubic & $10.8 \pm 0.1$ & $17.3 \pm 0.1$ \\
\hline \hline
\end{tabular}

${ }^{\mathrm{a}}$ Reference 20.

${ }^{\mathrm{b}}$ As explained in Ref. 13, a cofactor of 2 was missing in the experimental data of Ref. 20.

${ }^{\mathrm{c}}$ Reference 25 .

to be reliable at least qualitatively, despite the fact that we were unable to find experimental values. The calculations suggest that the FM domains in LSM are rather stable at low temperatures. Indirect support comes from the phase diagram in Ref. 7, which shows that LSM with a dopant fraction of $x=1 / 8$ is a ferromagnetic insulator at low temperature, and that antiferromagnetic structures are important only at low $\mathrm{Sr}$ concentrations. From the increased magnitudes of the calculated coupling constants for high-temperature LSM, one can expect that the stability of FM domains increases with temperature.

Based on a spectral ellipsometry, ${ }^{25}$ it was suggested recently that the LMO reveals low-energy $d$ - $d$ optical transition around $2 \mathrm{eV}[1.7 \mathrm{eV}$ (Ref. 26)], and $\mathrm{O}(2 p)-\mathrm{Mn}(3 d)$ charge transfer transition at $4.7 \mathrm{eV}$. This is in qualitative agreement with our Fig. 1, demonstrating projected density of states (PDOS) and indicating the band gap of $2.3 \mathrm{eV}$ between the top of the valence band (VB) consisting of a mixture of Mn $3 d$ and O $2 p$ states and the conduction band (CB) bottom formed mostly by Mn $3 d$ states. The main VB peak lying $\sim 2 \mathrm{eV}$ below consists almost of $\mathrm{O} 2 p$ states. Note that a fine structure of the VB, especially a weak 1.5-eV-wide subband around its top, depends on the exchange-correlation functional used: it arises mostly in B3LYP calculations and disappears in Hartree-Fock calculations. ${ }^{12}$

For both orthorhombic and pseudocubic LSM, our calculated PDOSs [Figs. 1(b) and 1(c)] are similar in the vicinity of the Fermi level. La or Sr contributions near the VB and/or $\mathrm{CB}$ regions are negligible. The hybridization of $\mathrm{Mn} 3 d / \mathrm{O} 2 p$ orbitals results in a semicovalent nature of the $\mathrm{Mn}-\mathrm{O}$ bond in manganite, which becomes more pronounced after $\mathrm{Sr}$ doping. The calculated Mn-O bond population (Mulliken analysis) has increased from $0.072 e$ in LMO to $0.084 e$ in LSM, confirming the increase in covalency.

Figures 1(b) and 1(c) demonstrate the half-metallic behavior of both LSM structures in their FM ground state. For $x=1 / 8$, one spin subband exhibits metallic character, while the other one is insulating, both in the low- and in the hightemperature phases. This behavior leads us to speculate that spin-polarized transport could be responsible for LSM elec-

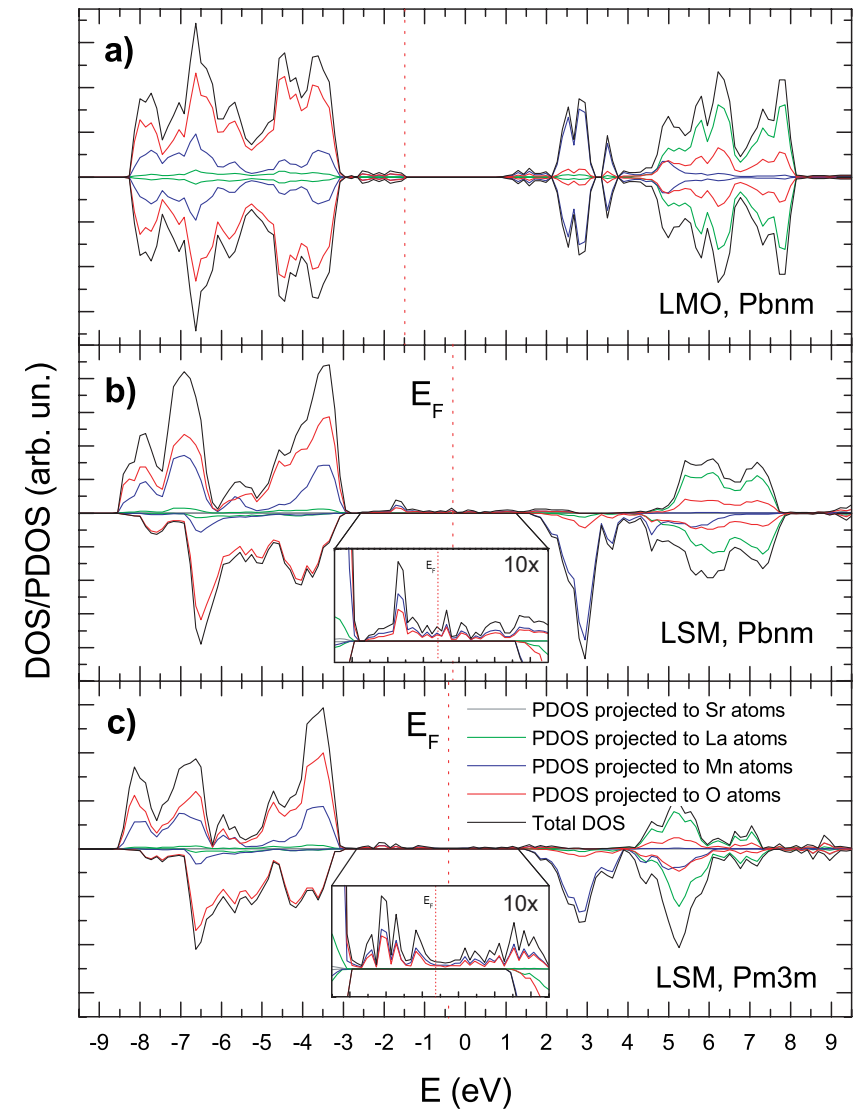

FIG. 1. (Color online) Total and projected DOS calculated for (a) orthorhombic LMO in A-AFM ground state, (b) orthorhombic LSM in FM ground state, and (c) cubic LSM in FM ground state. Positive DOS stands for spin-up electrons, negative for spin-down electrons. The energy scale is plotted relative to the vacuum level. The insets show DOS peaks in the gap magnified 10 times.

tronic conductivity. With increasing $\mathrm{Sr}$ doping, LSM is known to stabilize the $\mathrm{Mn}^{4+}$ valency relative to $\mathrm{Mn}^{3+}$. According to Ref. 5, disproportionation in $\mathrm{Mn}^{4+} / \mathrm{Mn}^{3+}$ is observed. Our Mulliken charge analysis shows that after $\mathrm{Sr}$ doping, a hole is relatively uniformly redistributed over the $\mathrm{Mn}-\mathrm{O}$ sublattice. The Mn charge is $1.81 e$ for LSM $(x$ $=1 / 8)$ in the FM ground state and $1.82 e$ in LMO; $-1.38 e$ and $-1.42 e$ for $\mathrm{O}$, respectively. Mulliken charges on La are practically unchanged upon doping.

In summary, we have performed DFT calculations with the B3LYP exchange-correlation functional of the magnetic and electronic structure of $\mathrm{La}_{0.875} \mathrm{Sr}_{0.125} \mathrm{MnO}_{3}$ and pure $\mathrm{LaMnO}_{3}$. The calculations show that, in agreement with previous calculations, LMO has an antiferromagnetically ordered ground state. LSM, on the other hand, has at low temperature a strong prevalence for a ferromagnetically ordered state. This prevalence for the ferromagnetic state is more pronounced as the one for the antiferromagnetic state in LMO and increases further in the high-temperature pseudocubic phase. Furthermore, we see, at least for the system sizes studied here, no indication of significant charge ordering around the Sr defect. Thus, we conclude from our calculations that LSM at high temperature is pseudocubic and prefers ferromagnetically ordered domains. Contrary to 
LMO, the DOS show half-metallic spin states in the band gap of LSM, which could be responsible for the electronic conductivity of LSM SOFC cathodes.

We are grateful for helpful discussions with S. Blügel and his group (IFF/FZJ). This work was supported by the Alexander von Humboldt Foundation, the "Fonds der Chemischen Industrie" (VCI), the MRSEC program of the NSF
(DMR-0520513) at the Materials Research Center of Northwestern University, the European Social Fund (ESF), and the Latvian Research Programme on Functional Materials and Technologies for Microelectronics and Photonics (Project No. 05.0005.1.1). Computer time by the John von Neumann Institute for Computing (VSR project id JIWV31) is gratefully acknowledged. *piskunov@lu.lv

${ }^{1}$ S. Taran, B. K. Chaudhuri, S. Chatterjee, H. D. Yang, S. Neeleshwar, and Y. Y. Chen, J. Appl. Phys. 98, 103903 (2005).

${ }^{2}$ J. Fleig, K. D. Kreuer, and J. Maier, in Handbook of Advanced Ceramics edited by S. Somiya, F. Aldinger, N. Claussen, R. M. Spriggs, K. Uchino, K. Koumoto, and M. Kaneno (Elsevier Academic, Amsterdam, 2003), Vol. II, pp. 59-105.

${ }^{3}$ F. Tietz, H. P. Buchkremer, and D. Stöver, J. Electroceram. 17, 701 (2006)

${ }^{4}$ D. J. Singh and W. E. Pickett, Phys. Rev. B 57, 88 (1998).

${ }^{5}$ G. Banach and W. M. Temmerman, Phys. Rev. B 69, 054427 (2004).

${ }^{6}$ H. Zenia, G. A. Gehring, G. Banach, and W. M. Temmerman, Phys. Rev. B 71, 024416 (2005).

${ }^{7}$ R. Klingeler, J. Geck, R. Gross, L. Pinsard-Gaudart, A. Revcolevschi, S. Uhlenbruck, and B. Büchner, Phys. Rev. B 65, 174404 (2002).

${ }^{8}$ J. Geck, P. Wochner, D. Bruns, B. Buchner, U. Gebhardt, S. Kiele, P. Reutler, and A. Revcolevschi, Phys. Rev. B 69, 104413 (2004).

${ }^{9}$ F. Mayr, C. Hartinger, and A. Loidl, Phys. Rev. B 72, 024425 (2005).

${ }^{10}$ S. Piskunov, E. Heifets, R. I. Eglitis, and G. Borstel, Comput. Mater. Sci. 29, 165 (2004).

${ }^{11}$ S. Piskunov, E. A. Kotomin, E. Heifets, J. Maier, R. I. Eglitis, and G. Borstel, Surf. Sci. 575, 75 (2005).

${ }^{12}$ D. Muñoz, N. M. Harrison, and F. Illas, Phys. Rev. B 69, 085115 (2004).

${ }^{13}$ R. A. Evarestov, E. A. Kotomin, Y. A. Mastrikov, D. Gryaznov,
E. Heifets, and J. Maier, Phys. Rev. B 72, 214411 (2005).

${ }^{14} \mathrm{R}$. Dovesi et al., CRYSTALO6 User's Manual (University of Torino, Torino, 2006), http://www.crystal.unito.it/

${ }^{15}$ M. D. Towler, N. L. Allan, N. M. Harrison, V. R. Saunders, W. C. Mackrodt, and E. Aprà, Phys. Rev. B 50, 5041 (1994).

${ }^{16}$ P. J. Hay and W. R. Wadt, J. Chem. Phys. 82, 299 (1984).

${ }^{17}$ M. Dolg, H. Stoll, A. Savin, and H. Preuss, Theor. Chim. Acta 75, 173 (1989).

${ }^{18}$ H. J. Monkhorst and J. D. Pack, Phys. Rev. B 13, 5188 (1976).

${ }^{19}$ T. Chatterji, B. Ouladdiaf, P. Mandal, B. Bandyopadhyay, and B. Ghosh, Phys. Rev. B 66, 054403 (2002).

${ }^{20}$ F. Moussa, M. Hennion, J. Rodriguez-Carvajal, H. Moudden, L. Pinsard, and A. Revcolevschi, Phys. Rev. B 54, 15149 (1996).

${ }^{21}$ J. Rodríguez-Carvajal, M. Hennion, F. Moussa, A. H. Moudden, L. Pinsard, and A. Revcolevschi, Phys. Rev. B 57, R3189 (1998).

${ }^{22}$ H. Hayashi, A. Sato, T. Azumi, Y. Udagawa, T. Inami, K. Ishii, and K. B. Garg, Phys. Rev. B 73, 134405 (2006).

${ }^{23}$ L. Pinsard, J. Rodríguez-Carvajal, and A. Revcolevschi, J. Alloys Compd. 262-263, 152 (1997).

${ }^{24}$ P. Reinhardt, I. Moreira, C. de Graaf, R. Dovesi, and F. Illas, Chem. Phys. Lett. 319, 625 (2000).

${ }^{25}$ N. N. Kovaleva, A. V. Boris, C. Bernhard, A. Kulakov, A. Pimenov, A. M. Balbashov, G. Khaliullin, and B. Keimer, Phys. Rev. Lett. 93, 147204 (2004).

${ }^{26}$ T. Saitoh, A. E. Bocquet, T. Mizokawa, H. Namatame, A. Fujimori, M. Abbate, Y. Takeda, and M. Takano, Phys. Rev. B 51, 13942 (1995). 